\title{
Volume Modulated Arc Therapy (VMAT) for pulmonary Stereotactic Body Radiotherapy (SBRT) in patients with lesions in close approximation to the chest wall
}

\section{Linda Ding ${ }^{1}$, Yuan-Chyuan Lo ${ }^{1}$, Sidney Kadish ${ }^{1}$, David Goff ${ }^{1}$, Richard S. Pieters ${ }^{1}$, Geoffrey Graeber ${ }^{2}$, Karl Uy ${ }^{2}$, Syed Quadri ${ }^{2}$, Richard Moser ${ }^{3}$, Kevin Martin ${ }^{4,5}$, John Day ${ }^{6}$ and Thomas J. FitzGerald ${ }^{1 *}$}

\author{
Department of Radiation Oncology, University of Massachusetts Medical School and the University of Massachusetts Memorial Health Care System, Worcester, \\ MA, USA \\ ${ }^{2}$ Department of Thoracic Surgery, University of Massachusetts Medical School and the University of Massachusetts Memorial Health Care System, Worcester, \\ MA, USA \\ ${ }^{3}$ Department of Neurosurgery, University of Massachusetts Medical School and the University of Massachusetts Memorial Health Care System, Worcester, MA, \\ USA \\ ${ }^{4}$ Department of Medicine, University of Massachusetts Medical School and the University of Massachusetts Memorial Health Care System, Worcester, MA, USA \\ ${ }^{5}$ Department of Medicine, St. Vincent Hospital, Worcester, MA, USA \\ ${ }^{6}$ Department of Medicine, Day Kimball Hospital, Putnam, CT, USA
}

\section{Edited by:}

Brian T. Collins, Georgetown University Hospital, USA

\section{Reviewed by:}

Sean Collins, Georgetown University Hospital, USA

Jaroslaw T. Hepel, Rhode Island Hospital/Brown University, USA

\section{*Correspondence:}

Thomas J. FitzGerald, Department of Radiation Oncology, University of Massachusetts Medical School and the University of Massachusetts Memorial Health Care System, 55 Lake Avenue North, Worcester, MA 01655, USA.

e-mail: thomas.fitzgerald@ umassmed.edu
Purpose: Chest wall pain and discomfort has been recognized as a significant late effect of radiation therapy in historical and modern treatment models. Stereotactic Body Radiotherapy (SBRT) is becoming an important treatment tool in oncology care for patients with intrathoracic lesions. For lesions in close approximation to the chest wall with motion management, SBRT techniques can deliver high dose to the chest wall. As an unintended target of consequence, there is possibility of imposing significant chest wall pain and discomfort as a late effect of therapy. The purpose of this paper is to evaluate the potential role of Volume Modulated Arc Therapy (VMAT) technologies in decreasing chest wall dose in SBRT treatment of pulmonary lesions in close approximation to the chest wall.

Materials and Methods: Ten patients with pulmonary lesions of various sizes and tomography in close approximation to the chest wall were selected for retrospective review. All volumes including tumor target, chest wall, ribs, and lung were contoured with maximal intensity projection maps and four-dimensional computer tomography planning. Radiation therapy planning consisted of static techniques including Intensity Modulated Radiation Therapy compared to VMAT therapy to a dose of $60 \mathrm{~Gy}$ in $12 \mathrm{~Gy}$ fraction dose. Dose volume histogram to rib, chest wall, and lung were compared between plans with statistical analysis.

Results: In all patients, dose and volume were improved to ribs and chest wall using VMAT technologies compared to static field techniques. On average, volume receiving $30 \mathrm{~Gy}$ to the chest wall was improved by $74 \%$; the ribs by $60 \%$. In only one patient did the VMAT treatment technique increase pulmonary volume receiving 20 Gy (V20).

Conclusions: VMAT technology has potential of limiting radiation dose to sensitive chest wall regions in patients with lesions in close approximation to this structure. This would also have potential value to lesions treated with SBRT in other body regions where targets abut critical structures.

Keywords: Volume Modulated Arc Therapy, radiation therapy, intrathoracic lesions, stereotactic body radiotherapy, chest wall

\section{INTRODUCTION}

Radiation therapy is an important treatment modality in cancer care. Acute normal tissue effects during therapy generally affect tissues of rapid self renewal potential including skin and mucosal surfaces. Late effects from treatment can affect tissues of both rapid and limited self renewal potential. Injuries to tissues of limited self renewal potential including bone, muscle, and nerve often become chronic in nature and result in significant pain with limited successful treatment options. In early breast cancer management, patients were treated with enface photon techniques with varied fractionation strategies including strategies not commonly employed in modern care. On occasion, these patients would develop chest wall injury and rib fracture (Dalinka et al., 1974; Overgaard, 1988). Even with more modern preIMRT breast cancer therapy techniques, areas of radiation dose inhomogeneity were identified in medial and lateral rib/chest 
wall/brachial plexus segments with the level of dose inhomogeneity exceeding $115 \%$ of prescription in significant volumes of the breast tissue and chest wall. Bone scans obtained at various time points from therapy would reveal activity in these locations and anecdotal rib fractures and chest wall soft tissue injury were seen in this population of patients. Intensity modulation has permitted improvement in radiation dose distribution in this population. Improvements in treatment technology including modern image guidance have re-established the role of accelerated treatment fractionation schedules in selected patients to strategic targets including lesions requiring motion management. Stereotactic radiosurgery (SRS) and radiation therapy have become important tools for modern care for the oncology patients. Both primary lesions and metastatic lesions in multiple body areas are successfully treated with radiosurgery and stereotactic radiation therapy. High fraction therapy to limited volumes may have a separate successful mechanism of tumor cell killing influencing the use of this form of therapy. High dose limited fraction radiation therapy used in SRS and SBRT is uniquely challenging when tumor target volumes lie in juxtaposition to normal tissue structures of limited self renewal potential such as chest wall, rib, and nerve. In this situation the risk of long-term injury is more likely. Chest wall injury and pain syndrome can be a significant and debilitating effect of therapy with limited treatment options once the injury becomes clinically apparent. There is increasing evidence that the risk of chest wall injury is related to the volume of chest wall receiving high dose radiation as an unintended target. Mutter and colleagues have recently demonstrated a significant increase in chest wall injury and pain syndrome driven by the volume of chest wall receiving 30 Gy (V30) (Mutter et al., 2012). Other investigators have demonstrated similar findings in retrospective review of radiosurgery treatment plans including issues associated with body habitus influencing treatment outcome (Voroney et al., 2009; Dunlap et al., 2010; Welsh et al., 2010).

In this paper, we evaluate the role of Volumetric Modulated Arc Therapy (VMAT) in limiting radiation dose to the chest wall volume in patients with pulmonary lesions in close approximation to the chest wall. The objective is to determine if this planning strategy may result in quantitative improvement of radiation dose to the chest wall in this important clinical situation.

\section{MATERIALS AND METHODS}

Ten patients with pulmonary lesions in close approximation to the chest wall (average $2 \mathrm{~cm}$ ) were chosen in retrospect from our patient population for this review as these patients appeared suitable for this analysis. Eight patients had primary disease of the lung (Stage 1, non-small cell lung cancer-NSCL) and two patients had metastatic disease with an average age at the time of treatment of 71 years (range 52-91). As part of the simulation process, all patients signed consent to permit use of their imaging and radiation therapy planning objects for education and research in a de-identified format.

\section{MOTION MANAGEMENT}

All patients underwent free breathing 4D computer tomography radiation therapy planning. For patients with motion less than $15 \mathrm{~mm}$, the clinical target volume (CTV) from the maximal intensity projection plus motion was chosen as the internal target volume (ITV). For patients with motion greater than $15 \mathrm{~mm}$, patients were planned with amplitude breathing defined and reproduced with cone beam CT validation of the target immediately prior to treatment execution. Planning target volume (PTV) was $2 \mathrm{~mm}$ for both planning strategies. Each patient was fitted with a Vac-loc device (Culver City, IA) fitted with a wingboard for radiation therapy planning and treatment.

\section{RADIATION THERAPY PLANNING}

For static field planning, patients were planned using 50\% IMRT beam strategy and 50\% static field. On average, seven fields were used for treatment plans using static fields. For VMAT planning, two one-half arcs were used and directed to the same target volumes.

Optimization constraints included 40 Gy to less than $10 \%$ of the chest wall/ribs and 20 Gy to less than $20 \%$ of the affected lung volume or better. The constraints were identical for both planning strategies.

\section{RADIATION THERAPY DOSE}

All patients were planned to receive 60 Gy in 12 Gy fractions used by both treatment plans for purpose of comparison.

\section{NORMAL TISSUE STRUCTURES}

The chest wall was drawn from the inner surface of the rib and included $2 \mathrm{~cm}$ beyond this point from the sternum to the facet joint of the vertebral body. The rib was drawn as a separate target by using $4 \mathrm{D}$ CT in maximum intensity projection. The lung was drawn as a separate target. The V20 was calculated relative to the entire lung volume in the affected lung and compared between each plan. The V30 of the chest wall and rib was compared between the two plans for each patient.

\section{STATISTICS}

Student $T$-test was used to compare difference in V30 for the rib and chest wall and the pulmonary V20 between both plans for each patient.

\section{RESULTS}

Patient demographics, CTV/PTV volumes, and distance of the target from the chest wall are listed in Table 1. The results for the V30 to the chest wall and ribs as well as the V20 to the pulmonary parenchyma are demonstrated in Table 2 . The absolute volumes of rib, chest wall, and pulmonary parenchyma are listed in Tables 3a,b,c, respectively. Individual patient maximum tumor motion is listed in Table 4. All patients demonstrated improvement in V30 for the chest wall and rib using VMAT planning. The average improvement to the chest wall was $74.3 \%$ and to the ribs $60.8 \%$ using the VMAT plan $(p<0.05)$. Only one patient demonstrated an increase in pulmonary V20 with the VMAT plan.

\section{DISCUSSION}

Pain and discomfort of the chest wall can be a late effect of radiation management and treatment of this effect can be unsatisfactory for both the patient and the physician. Often treatment 
Table 1 | Patient demographics and tumor statistics.

\begin{tabular}{lccll}
\hline $\begin{array}{l}\text { Patient (age } \\
\text { during treatment } \\
\text { time) }\end{array}$ & CTV(cc) & PTV(cc) & Tumor type & $\begin{array}{l}\text { Distance to the } \\
\text { closest rib (cm) }\end{array}$ \\
\hline 1 (91) & 38 & 74.3 & NSCL & 0.8 \\
2 (77) & 17.9 & 43.3 & $\begin{array}{l}\text { Metastasis/ } \\
\text { breast }\end{array}$ & 2.2 \\
& & & NSCL & 1.5 \\
$3(80)$ & 16.1 & 49.8 & NSCL & 2.5 \\
$4(74)$ & 14.3 & 74.8 & NSCL & 2.4 \\
$5(62)$ & 11.4 & 35.1 & NSLC & 2.8 \\
$6(64)$ & 1.7 & 14.7 & Metastasis/ & 1.0 \\
7 (62) & 0.9 & 9 & colon & \\
& & & NSCL & 1.8 \\
8 (52) & 9.2 & 38.5 & NSCL & 2.0 \\
9 (84) & 25.6 & 47 & NSCL & 2.3 \\
$10(61)$ & 4.7 & 18 & & 1.9 \\
Average (71 years & 14.0 & 40.4 & & \\
old) & & & &
\end{tabular}

Table 2 | V30 for chest wall and ribs was reduced for all 10 patients from original planning technique.

\begin{tabular}{llll}
\hline Patient & $\begin{array}{l}\text { Chest wall volume } \\
\text { receive } \mathbf{3 0 ~ G y ~ ( \% ) ~}\end{array}$ & $\begin{array}{l}\text { Ribs receive } \\
\mathbf{3 0} \mathbf{~ G y}(\%)\end{array}$ & V20 lung dose (\%) \\
\hline 1 & -65.5 & -59.2 & -2.4 \\
2 & -60.5 & -91.1 & -32.0 \\
3 & -32.2 & -11.1 & -6.5 \\
4 & -93.3 & -51.4 & 12.6 \\
5 & -55.0 & -47.9 & -18.3 \\
6 & -57.0 & -100.0 & -54.1 \\
7 & -100.0 & -69.1 & -26.3 \\
8 & -62.1 & -37.1 & -3.9 \\
9 & -54.1 & -40.9 & -16.7 \\
10 & -55.6 & -100.0 & -52.0 \\
\hline
\end{tabular}

V20 lung dose decreased for 9 of the 10 patients. The data is presented as the percent decrease in volume treated using VMAT technology.

Table 3a | Rib volume receiving $30 \mathrm{~Gy}$.

\begin{tabular}{lcc}
\hline Patient no. & Hybrid SBRT (cc) & RA SBRT (cc) \\
\hline 1 & 38.3 & 15.6 \\
2 & 5.6 & 0.5 \\
3 & 10.2 & 9.0 \\
4 & 17.3 & 8.4 \\
5 & 19.9 & 10.4 \\
6 & 3.1 & 0.0 \\
7 & 3.3 & 1.0 \\
8 & 15.1 & 9.5 \\
9 & 14.1 & 8.4 \\
10 & 2.7 & 0 \\
Average & 13.0 & 6.3
\end{tabular}

Table $3 \mathrm{~b}$ | Chest wall $(2 \mathrm{~cm})$ volume receiving $30 \mathrm{~Gy}$.

\begin{tabular}{lcc}
\hline Patient no. & Hybrid IMRT SBRT (cc) & RA SBRT (cc) \\
\hline 1 & 501.6 & 139.7 \\
2 & 30 & 3.1 \\
3 & 134.1 & 65.7 \\
4 & 347 & 56.3 \\
5 & 330.6 & 56 \\
6 & 56.7 & 0 \\
7 & 17.1 & 7.7 \\
8 & 247.7 & 55.8 \\
9 & 219.2 & 72 \\
10 & 55.4 & 20 \\
Average & 193.9 & 47.6 \\
\hline
\end{tabular}

Table 3c | Percent lung volume receiving $20 \mathrm{~Gy}$.

\begin{tabular}{lcc}
\hline Patient no. & Hybrid IMRT SBRT (\%) & RA SBRT (\%) \\
\hline 1 & 12.3 & 12.0 \\
2 & 22.5 & 15.3 \\
3 & 13.9 & 13.0 \\
4 & 8.0 & 6.5 \\
5 & 3.8 & 2.8 \\
6 & 17.6 & 19.8 \\
7 & 9.8 & 4.5 \\
8 & 15.4 & 14.8 \\
9 & 8.4 & 7.0 \\
10 & 12.3 & 5.9 \\
Average & 12.4 & 10.2 \\
\hline
\end{tabular}

Table 4 | Maximum tumor motion measured on 4D CT.

\begin{tabular}{lllllllllll}
\hline Patient no. & $\mathbf{1}$ & $\mathbf{2}$ & $\mathbf{3}$ & $\mathbf{4}$ & $\mathbf{5}$ & $\mathbf{6}$ & $\mathbf{7}$ & $\mathbf{8}$ & $\mathbf{9}$ & $\mathbf{1 0}$ \\
\hline Motion $(\mathrm{mm})$ & 8.5 & 9 & 5 & 3 & 9.4 & 12.4 & 10.8 & 9.8 & 2 & 3
\end{tabular}

management options are limited to analgesia and nerve blocks, each of which is often partially effective.

Traditional radiation therapy fractionation regimens can be associated with the development of discomfort in the chest wall and in more historical models rib fractures were identified on occasion as part of breast cancer management (Dalinka et al., 1974; Overgaard, 1988; Pierce et al., 1992; Hall, 2000; Jackson et al., 2010). More modern radiation therapy treatment planning strategies including volumetric treatment planning with intensity modulation radiation therapy treatment execution permit thorough examination of the radiation therapy dose to chest wall structures, often permitting planning strategies to limit the volume and extent of radiation dose inhomogeneity with the chest wall target. These advances, including the use of integrated advanced technology imaging techniques with real time target validation, have permitted the field of radiation oncology to re-visit the use of high dose limited fractionation treatment schedules including stereotactic therapy for multiple body regions and tumor targets. For tumor targets in close approximation to 
the chest wall, investigators are recognizing there is risk of injury to the chest wall with accelerated fraction radiation therapy, especially when high volumes of the chest wall receive greater than $30 \mathrm{~Gy}$. One series suggests that the risk of injury to the chest wall with radiosurgery techniques is greater than $40 \%$ when lesions are within $2 \mathrm{~cm}$ of the chest wall (Asai et al., 2012). Therefore, when the tumor target is in close approximation to the chest wall, there would be a higher risk using more traditional radiotherapy technologies that use static fields as there would be more rib and chest wall volume in the therapy field (Dalinka et al., 1974; Overgaard, 1988; Pierce et al., 1992; Meric et al., 2002; Shioyama et al., 2005; Zimmerman et al., 2006; Kyas et al., 2007; Baumann et al., 2009; Petersson et al., 2009; Siva et al., 2010; Andolino et al., 2011; Nambu et al., 2011; Stephans et al., 2012). This risk maybe further exaggerated with larger PTVs needed for set up uncertainty indicating the importance of immobilization and treatment reproducibility. In our experience, we have found that personalized Vac-Loc with integrated wingboard works well for daily reproducibility of patient set up coupled with shallow breathing techniques. We have found that multiple compression devices have introduced additional error into the patient set up and have not demonstrated an advantage in patient care or reproducibility of each treatment. We identify the breathing cycle amplitude during the simulation process and have successfully reproduced the target with cone beam CT validation by re-establishing the breathing amplitude cycle used at the time of simulation. In patients with significant motion or change in respiratory status, we perform a second simulation to validate that the ITV is identical to the volume established at the time of primary simulation. To date, no patient has sustained a chest wall injury and none have relapsed in the local target volume.

The use of modulated arc radiotherapy appears in our study to have significant potential benefit for patient care in the situation where tumor comes in close approximation to the chest wall region. VMAT can accommodate the multiple sloping surfaces of the chest wall and the dynamic nature of the simultaneous dual motion of both the gantry and multileaf collimators permits potentially more optimal radiation therapy treatment planning and therapy execution. VMAT technology may decrease the risk of injury to this selected patient population. In our study, significant decreases in radiation dose to both chest wall and rib targets were seen in side by side comparison between VMAT technology and traditional SBRT treatment plans with static fields. Figures 1 and 2 demonstrate the improvement achievable in supine and prone positions, respectively. In our analysis the average number of fields was seven (7) for non-VMAT planning. Another

\section{VMAT Technology for Pulmonary SBRT in Patients with Lesions in Close Approximation to the Chest Wall}

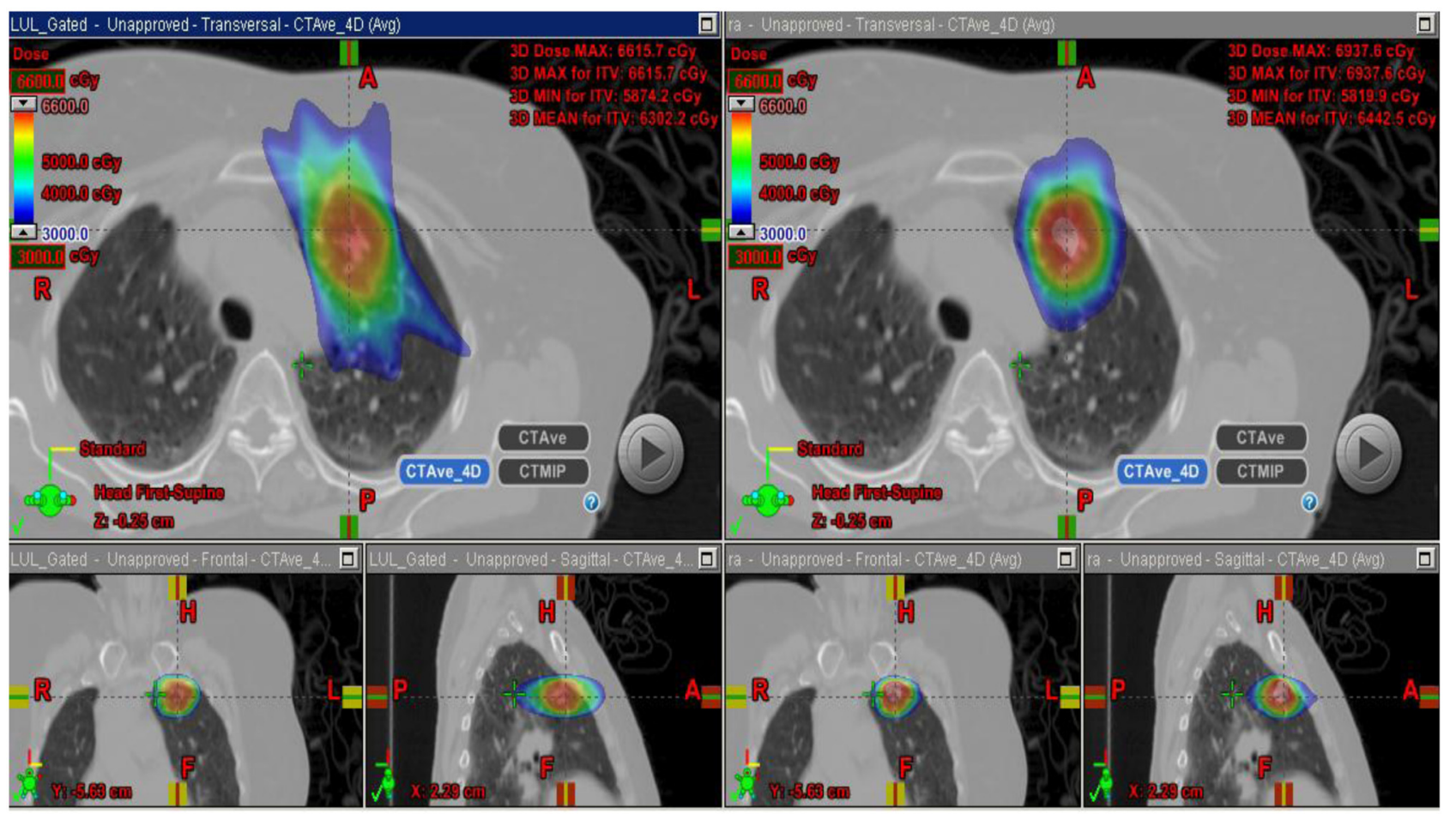

FIGURE 1 | Demonstrates improvement in dosimetry in a lesion in the anterior segment of the left upper lobe with the static field plan on the left and the VMAT plan on the right. 


\section{VMAT Technology for Pulmonary SBRT in Patients with Lesions in Close Approximation to the Chest Wall}

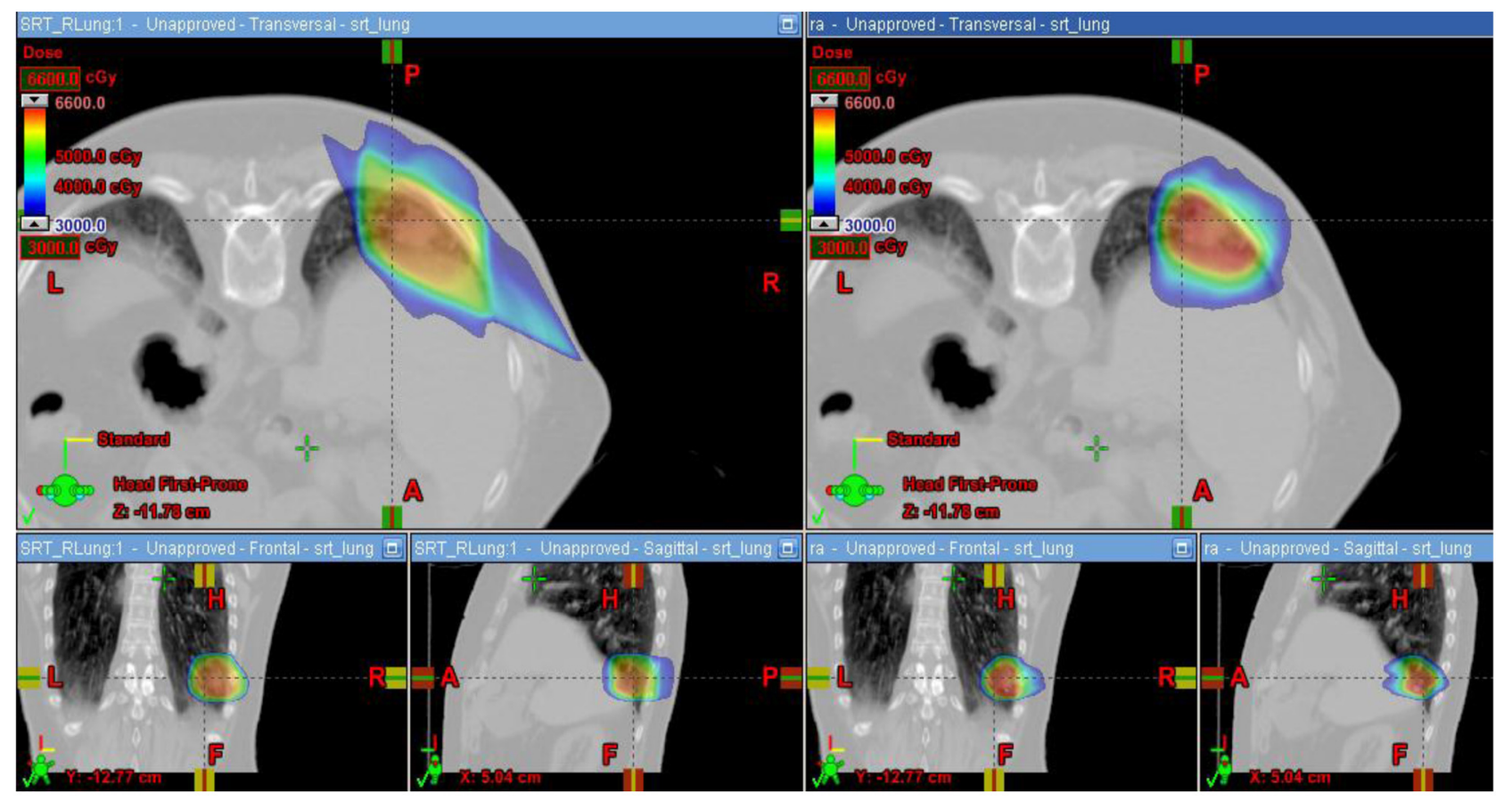

FIGURE 2 | Demonstrates improvement in dosimetry to the chest wall and ribs in a patient in the prone position with a lesion in the posterior segment of the right lower lobe. The static field plan is on the left and the VMAT plan is on the right.

advantage to VMAT therapy is speed of treatment delivery and subsequent improvement in therapy dose rate. Traditional static field radiosurgery treatment techniques with multiple fields can take a significant amount of time to complete, often greater than 30 min coupled with time for image validation. VMAT therapy can take place in a much more abbreviated time frame (a few min) further decreasing risk of target motion during therapy as well as provide a more comfortable treatment environment for the patient. The primary concern of arc therapy is in this population that arc treatment will increase dose to pulmonary parenchyma in patients treated with this technique. We note only one patient experienced an increase in pulmonary V20 with optimal VMAT planning relative to planning with static fields (patient $4 / 12 \%$ increase). It is likely VMAT technology can play an important role in hepatic radiotherapy when tumor comes in close approximation to the chest wall as well as other tumor targets in multiple body locations that abut critical normal tissue structures.

\section{REFERENCES}

Andolino, D., Forquer, J., Henderson, M., Barriger, R., Shapiro, R., Brabham, J., et al. (2011). Chest wall toxicity after stereotactic body radiotherapy for malignant lesions of the lung and liver. Int. J. Radiat. Oncol. Biol. Phys. 80, 692-697.

Asai, K., Shioyama, Y., Nakamura, K., Sasaki, T., Ohga, S., Nonoshita, T., et al. (2012). Radiation induced rib fractures after hypofractionated stereotactic body radiation therapy: risk factors and dose volume relationship. Int. J. Radiat. Oncol. Biol. Phys. 84, 768-773.
Baumann, P., Nyman, J., Hoyer, M., Wennberg, B., Gagliardi, G., Lax, I., et al. (2009). Outcome in a prospective phase 2 trial of medically inoperable stage 1 non-small cell carcinoma lung cancer patients treated with stereotactic body radiotherapy. J. Clin. Oncol. 27, 3290-3298.

Dalinka, M., Edeikeri, J., and Finkelstein, J. (1974). Complications of radiation therapy: adult bone. Semin. Roentgenol. 9, 29-40.

Dunlap, N. E., Cai, J., Biedermann, G. B., Yang, W., Benedict, S. H., Sheng,
K., et al. (2010). Chest wall volume receiving $>30$ Gy predicts risk of severe pain and/or rib fracture after stereotactic body radiotherapy. Int. J. Radiat. Oncol. Biol. Phys. 76, 796-801.

Hall, E. J. (2000). Radiobiology for the Radiologist. 5th Edn. Philadelphia: Lippincott, Williams, and Wilkins.

Jackson, A., Marks, L. B., Bentzen, S. M., Eisbruch, A., Yorke, E. D., Ten Haken, R. K., et al. (2010). The lessons of QUANTEC: Recommendations for reporting and gathering data on dose-volume dependencies of treatment outcome. Int. J. Radiat. Oncol. Biol. Phys. 76, S155-S160.

Kyas, I., Hof, H., Debus, J., Schlegel, W., and Karger, C. (2007). Prediction of radiation induced changes in the lung after stereotactic body radiation therapy of non small cell lung cancer. Int. J. Radiat. Oncol. Biol. Phys. 67, 768-774.

Meric, F., Buchholtz, T., Murza, N., Vlastos, G., Ames, F., Ross, M., et al. (2002). Long term complications associated with breast conservation surgery and radiotherapy. Ann. Surg. Oncol. 9, 543-548. 
Mutter, R., Liu, F., Abreu, A., Yorke, E., Jackson, A., and Rosenzweig, K. E. (2012). Dose-volume parameters predict for the development of chest wall pain after stereotactic body radiation for lung cancer. Int. J. Radiat. Oncol. Biol. Phys. 82, 1783-1790.

Nambu, A., Onshi, H., Aoki, T., Koshishi, K., Komiyama, T., Marino, K., et al. (2011). Rib fracture after stereotactic radiotherapy on follow up thin section computer tomography in 177 primary lung cancer patients. Radiat. Oncol. 6, 137-142.

Overgaard, M. (1988). Spontaneous radiation induced rib fractures in breast cancer patients treated with post-mastectomy irradiation: a clinical radiobiological analysis of the influence of fraction size and dose-response relationships on late bone damage. Acta Oncol. 27, 117-122.

Petersson, N., Nyman, J., and Johnasson, K. (2009). Radiation induced rib fractures after hypofractionated stereotactic body radiation of non small cell lung cancer. A dose-volume response analysis. Radiother. Oncol. 91, 360-368.

Pierce, S., Recht, A., Lingos, T., Abner, A., Vicini, F., Silver, B., et al. (1992). Long term complications associated with conservative surgery and radiation therapy in patients with early stage breast cancer. Int. J. Radiat. Oncol. Biol. Phys. 23, 915-923.

Shioyama, Y., Nakayama, K., Anai, S., Sasaki, T., Ooga, S., Saku, M., et al. (2005). Stereotactic radiotherapy for lung and liver tumors using a body cast system. Setup accuracy and preliminary clinical outcome. Radiat. Med. 23, 407-413.

Siva, S., MacManus, M., and Ball, D. (2010). Stereotactic radiotherapy for pulmonary oligometastasis. A systemic review. J. Thorac. Oncol. 5, 1091-1098.

Stephans, K., Djemil, T., Tendulkar, R., Robinson, C., Reddy, C., and Videtic, G. (2012). Prediction of chest wall toxicity from lung stereotactic body radiotherapy (SBRT).
Int. J. Radiat. Oncol. Biol. Phys. 82, 974-980.

Voroney, J. P., Hope, A., Dahle, M. R., Purdie, T. G., Franks, K. N., Pearson, S., et al. (2009). Chest wall pain and rib fracture after stereotactic radiotherapy for peripheral non small cell lung cancer. J. Thorac. Oncol. 4, 1035-1037.

Welsh, J., Thomas, J., Shah, D., Allen, P. K., Wei, X., Mitchell, K., et al. (2010). Obesity increases the risk of chest wall pain from thoracic stereotactic body radiation therapy. Int. J. Radiat. Oncol. Biol. Phys. 81, 91-96.

Zimmerman, F., Geinitz, H., Schill, S., Thamm, R., Neider, C., Schratzenstaller, U., et al. (2006). Stereotactic hypofractionated radiotherapy in stage 1 (T1-2 N0M0) non small cell lung cancer (NSCLC). Acta Oncol. 45, 796-801.

Conflict of Interest Statement: The authors declare that the research was conducted in the absence of any commercial or financial relationships that could be construed as a potential conflict of interest.

Received: 10 August 2012; accepted: 14 January 2013; published online: 22 February 2013.

Citation: Ding L, Lo $Y$-C, Kadish S, Goff D, Pieters RS, Graeber G, Uy K, Quadri S, Moser R, Martin K, Day J and FitzGerald TJ (2013) Volume Modulated Arc Therapy (VMAT) for pulmonary Stereotactic Body Radiotherapy (SBRT) in patients with lesions in close approximation to the chest wall. Front. Oncol. 3:12. doi: 10.3389/fonc.2013.00012

This article was submitted to Frontiers in Radiation Oncology, a specialty of Frontiers in Oncology.

Copyright (c) 2013 Ding, Lo, Kadish, Goff, Pieters, Graeber, Uy, Quadri, Moser, Martin, Day and FitzGerald. This is an open-access article distributed under the terms of the Creative Commons Attribution License, which permits use, distribution and reproduction in other forums, provided the original authors and source are credited and subject to any copyright notices concerning any third-party graphics etc. 